\title{
Abnormal keratocytes and stromal inflammation in chronic phase of severe ocular surface diseases with stem cell deficiency
}

\author{
T Saito, ${ }^{1}$ K Nishida, ${ }^{2}$ H Sugiyama, ${ }^{1}$ M Yamato, ${ }^{3}$ N Maeda, ${ }^{1}$ T Okano, ${ }^{3}$ Y Tano ${ }^{1}$
}

${ }^{1}$ Department of Ophthalmology, Osaka University Medical School, Suita, Japan;

${ }^{2}$ Department of Ophthalmology, Tohoku University School of

Medicine, Sendai, Japan;

${ }^{3}$ Institute of Advanced

Biomedical Engineering and

Science, Tokyo Women's

Medical University, Tokyo,

Japan

Correspondence to:

Dr K Nishida, PhD, Department

of Ophthalmology, Tohoku

University School of Medicine,

1-1 Seiryomachi, Aoba-ku,

Sendai 980-8574, Japan;

knishida@oph.med.tohoku.ac.jp

Accepted 4 November 2007

Published Online First

22 January 2008

\begin{abstract}
Background/Aims: Stevens-Johnson syndrome (SJS), ocular cicatricial pemphigoid (OCP) and alkali burns are associated with chronic, severe inflammation of the ocular surface that occasionally lead to corneal stem cell deficiencies. The corneal stroma in these diseases has not been studied comprehensively. The purpose of this study was to determine whether the keratocytes in the stroma were normal and whether the stroma remained inflamed in the chronic phase of these diseases.
\end{abstract}

Methods: Five pathological corneas, two with SJS, two with OCP and one with an alkali burn were examined. Corneal specimens were obtained during lamellar keratoplasty and the histological sections were immunostained with antibodies against CD34 and several cell surface antigens. The level of expression of proteoglycans (lumican, keratocan, biglycan) and chemokines (monocyte chemoattractant protein 1, macrophage inflammatory protein (MIP) $1 \alpha$, MIP1 $\beta$ ) were examined by quantitative real-time RT-PCR.

Results: The number of CD34-positive cells in the stroma was decreased and the expression level of biglycan increased in all of the pathological corneas. The numbers of CD45-positive and CD14-positive cells were increased in four of the five pathological corneas. The expression level of MIP1 $\alpha$ and MIP1 $\beta$ were markedly increased in all of the pathological corneas.

Conclusions: These findings indicate that the keratocytes are abnormal and inflammation is still present in the corneal stroma in the chronic phase of SJS, OCP and alkali burns.

Stevens-Johnson syndrome (SJS) and ocular cicatricial pemphigoid (OCP) of the cornea occasionally result in total limbal stem cell deficiency that often leads to severe ocular complications, which do not respond to classic treatment either in the acute phase or in the chronic phase. ${ }^{1-4}$ The outcome of surgery for these diseases is also poor, ${ }^{5}$ suggesting that the prolonged inflammation and severe dry eye or abnormal epithelial differentiation is not conducive to good surgical results. ${ }^{6}$

Severe alkali burns of the cornea also result in limbal stem cell deficiency and poor prognosis in some cases. $^{7-10}$ Compared with immunologically driven diseases such as SJS, however, the operative outcome is reported to be better especially when an autograft limbal transplantation is performed. ${ }^{11-13}$

In the chronic phase of SJS and chemical injury, inflammatory cells infiltrate into the conjunctivalised corneal epithelium. ${ }^{14}$ The outer layer of the corneal stroma under a pannus is occasionally invaded by blood vessels and opacities develop in these areas, but the pathological alterations in these areas have not been studied in detail. We hypothesised that abnormalities of keratocytes and persistent inflammation exist in the corneal stroma during the chronic phase of ocular surface diseases. To determine the pathological state of corneal stroma of eyes with severe ocular surface diseases covered by a pannus in the chronic phase, we examined five pathological corneas. If our hypothesis is correct, it should be possible to alter the environment around the corneal stroma to improve the surgical results.

\section{MATERIALS AND METHODS}

\section{Patients and normal corneas}

The procedures used conformed to the tenets of the Declaration of Helsinki and were approved by the Institutional Review Board of Osaka University Hospital. After obtaining informed consent, five pathological corneas were obtained from patients undergoing lamellar keratoplasty at the Department of Ophthalmology, Osaka University Hospital, from August 2005 to February 2006. Patients' profiles are presented in table 1 and slit-lamp photographs are shown in fig 1. During surgery, the pannus was removed with microforceps and a corneal button was removed with a $7 \mathrm{~mm}$ or $7.5 \mathrm{~mm}$ trephine and treated within one hour after excision. Six normal corneas were obtained from an eye bank in the United States and kept in Optisol GS storage medium (Bausch and Lomb, Rochester, New York, USA) at $4^{\circ} \mathrm{C}$. An $8 \mathrm{~mm}$ trephine was used to remove the corneal button and the buttons were processed on postmortem days 4 to 5 .

The pathological and normal corneas were cut into three pieces: one-third was embedded in optimal cutting temperature compound and cut on a cryostat at a thickness of $10 \mu \mathrm{m}$; the second third was embedded in paraffin and cut at $5 \mu \mathrm{m}$; and the last third was used for RNA analyses.

\section{Immunohistochemical staining}

The cryosections were fixed in cold acetone and processed for the detection of CD34, CD68, CD4, CD8, CD19, and HLA-DR and other sections were fixed in $1 \%$ paraformaldehyde for CD45 and CD14. ${ }^{15}$ To eliminate non-specific immune staining, the sections were exposed to $5 \%$ normal mouse serum when staining with CD34, CD4, CD8, and CD19 antibodies. Anti-Fc receptor blocker and isotype-matched immunoglobulin for each antibody were used in the staining of CD45, CD14, CD68, and HLA-DR. ${ }^{16}$ online under the BMJ Journals unlocked scheme, see http:// bjo.bmi.com/info/unlocked.dtl 
Table 1 Patients' clinical data

\begin{tabular}{lllllll}
\hline & Age (years) & Gender & VA & Duration (years) & Stromal opacity & Inflammatory cells \\
\hline SJS1 & 57 & F & 0.04 & 12.5 & Mild & - \\
SJS2 & 68 & F & 0.01 & 25 & Mild & - \\
OCP1 & 70 & F & 0.03 & - & Moderate-severe & + \\
OCP2 & 75 & M & 0.02 & - & Moderate & - \\
Alkali burn & 26 & M & HM & 6.5 & Severe & + \\
\hline
\end{tabular}

Age, age at surgery; Duration, duration from initial attack (Stevens-Johnson syndrome; SJS) or injury (alkali burn) to surgery; HM, hand movement; Inflammatory cells: +, recognised infiltration in haematoxylin and eosin (HE) stained section; - , not recognised infiltration in HE section; $\mathrm{OCP}$, ocular cicatricial pemphigoid; VA, visual acuity at the time of surgery.

All specimens were incubated with primary antibodies at $4^{\circ} \mathrm{C}$ overnight at the dilution shown in table 2. For negative controls, normal mouse immunoglobulin G ( $\operatorname{IgG}$ ) was used instead of the primary antibodies. Direct staining was performed for CD45 and CD14. For the other antigens, biotinylated donkey anti-mouse IgG (Jackson ImmunoResearch Laboratories, West Grove, Pennsylvania, USA; 1 : 200) was used as the secondary antibody and specimens were incubated for one hour followed by incubation in fluorescein isothiocyanate conjugated streptavidin conjugate (Zymed Laboratories, San Francisco, California, USA; 1 : 400) for one hour at room temperature. The nuclei of all sections were stained with Hoechst33342 (Sigma-Aldrich Corporation, St Louis, Missouri, USA; $1: 400$ ) and the sections were examined with a fluorescence microscope (Carl Zeiss, Tokyo, Japan) or a confocal microscope (Olympus, Tokyo, Japan).

To count the CD45-positive cells, sections from the central area, paracentral area (14 to 48 sections from the central sections) and peripheral area of the corneal button were used. For pathological corneas, CD45-positive and all cells in all fields for each area in a representative section were counted. For normal corneas, the number of CD45-positive and all cells in a $20 \times$ objective field (upper part of stroma) were counted. CD45positive and all cells in two fields per section were counted and the average number was used. The percentage of CD45-positive cells was determined by dividing the number of CD45-positive cells by the total number of all cells in each section. For normal corneal stroma, data are reported as the average of three corneas.

\section{RNA preparation}

For the normal corneal buttons, the epithelium was carefully removed from the stroma by scraping the outer surface of the cornea, whereas the endothelium and Descemet's membrane were peeled away with fine forceps. Residual pannus was removed with microforceps or was scraped off. Then the stromas were placed in RNA later (Qiagen Inc, Valencia, California, USA) until used and cut into 10 pieces. They were homogenised and the RNA was purified according to the EZ1 RNA Universal Tissue Kit (Qiagen) protocol.

\section{cDNA preparation and quantitative real-time RT-PCR analysis}

Reverse transcription was performed using the SuperScript First-Strand Synthesis System for RT-PCR (Invitrogen, Carlsbad, California, USA), according to the manufacturer's protocol and complimentary DNA was used as a template for PCR. Quantitative real-time RT-PCR was carried out using the ABI Prism 7900HT Sequence Detection System (Applied Biosystems Inc, Foster City, California, USA) according to the manufacturer's protocol. Primer pairs and TaqMan MGB probes labelled with 6-carboxyfluorescein at the 5'-end and nonfluorescent quencher at the $3^{\prime}$-end, were purchased from commercial sources (Applied Biosystems).

Thermocycling used 45 cycles at $95^{\circ} \mathrm{C}$ for 15 seconds and $60^{\circ} \mathrm{C}$ for one minute with an initial cycle at $50^{\circ} \mathrm{C}$ for two minutes and $95^{\circ} \mathrm{C}$ for 10 minutes. For negative controls, non-reverse transcribed total RNA was used as a template for all experiments. All assays were run in duplicate. The gene expression was normalised to that of glyceraldehyde-3-phosphate dehydrogenase in each sample.

\section{RESULTS}

\section{Corneal opacity and microscopic examination}

Slit-lamp examination before surgery showed that all of the patients had no clinical evidence of inflammation on the ocular surface, such as conjunctival injection, corneal infiltration, or oedema. However, they all had corneal opacities of different grades (fig 1, table 1). The corneal opacity of the SJS2 cornea appeared mildest under a dissecting microscope. The OCP1 and alkali burned corneas had denser stromal opacities and HE stained sections showed an accumulation of cells with dark round nuclei, which were different from keratocytes whose

Table 2 Primary antibodies used for immunohistochemistry

\begin{tabular}{llcl}
\hline Antibody (clone) & Isotype & Dilution & Specificity \\
\hline CD34 classll (QBEnd-10) & Mouse IgG1 & $1 / 40$ & Keratocytes \\
CD45-FITC (2D1) & Mouse IgG1 & $1 / 20$ & Panleukocytes \\
CD14-PE (M5E2) & Mouse IgG2a & $1 / 5$ & Monocytes, macrophages, DC, LC \\
CD68 (KP1) & Mouse IgG1 & $1 / 100$ & Monocytes or macrophages, DC, \\
CD4 (BC/1F6) & & & granulocytes, myeloid progenitor cells \\
CD8 (UCH-T4) & Mouse IgG1 & $1 / 10$ & T lymphocytes \\
CD19 (HD37) & Mouse IgG2a & $1 / 100$ & Cytotoxic/suppressor T lymphocytes \\
HLA-DR (LN3) & Mouse IgG1 & $1 / 100$ & Mature and immature B cells \\
\hline
\end{tabular}

DC, dendritic cell; FITC, fluorescein isothiocyanate conjugated; IgG, immunoglobulin G; LC, Langerhans cell; PE, phycoerythrin. Monoclonal antibody sources: CD34 class II and CD19, Dako Cytomation, Carpinteria, California, USA; CD45-FITC and CD14-PE, BD Biosciences, San Jose, California, USA; CD68 and CD4, Abcam Ltd, Cambridge, UK; CD8, Chemicon, Temecula, California, USA; HLA-DR, Lab Vision Corporation, Fremont, California, USA. 
Figure 1 Photographs of eyes of patients before and during the surgery. Haematoxylin and eosin (HE) sections of excised corneal stroma are also shown. No evidence of inflammation was seen preoperatively. Intraoperative photographs show corneal stroma after pannus was removed. The severity of the opacity varied among patients. In the HE sections, ocular cicatricial pemphigoid (OCP) type 1 and alkali burned corneas show an accumulation of inflammatory cells. The thickness of the corneal button in HE sections varied from 130 to $380 \mu \mathrm{m}$ (bar $=100 \mu \mathrm{m})$. SJS, StevensJohnson syndrome.

Informed consent was obtained for publication of this figure.
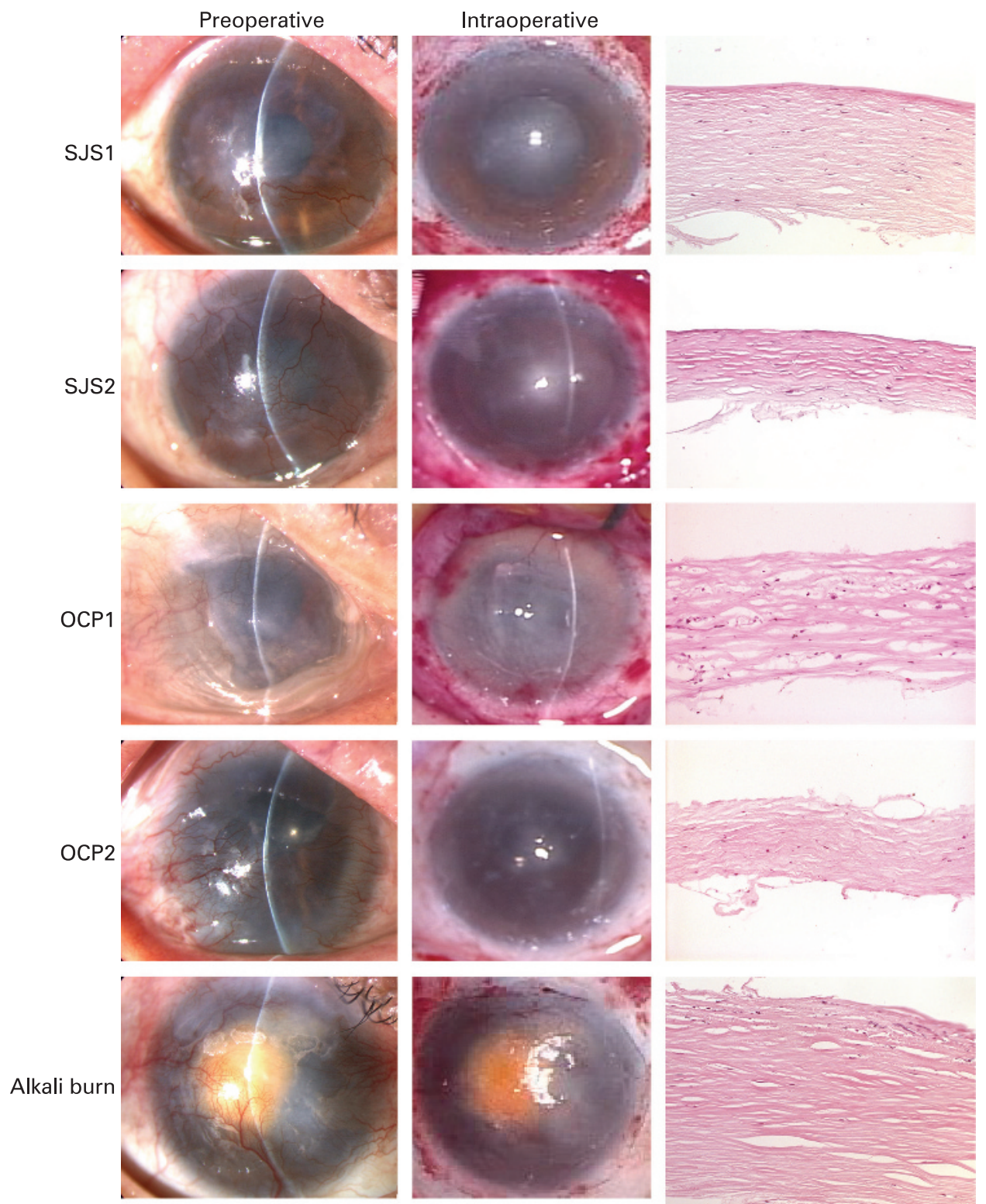

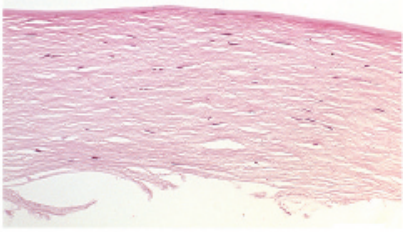

एᄆ

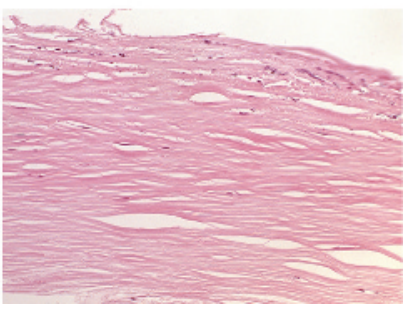

nuclei were flattened. The accumulated nuclei were considered to be those of inflammatory cells. The keratocytes were not uniformly distributed.

\section{Distribution of CD34-positive keratocytes in normal and pathological corneas}

The anti-CD34 class 2 antibody recognises normal keratocytes. ${ }^{17}{ }^{18}$ In the normal corneas, keratocytes showing strong CD34 immunoreactivity were seen throughout the stroma. In the four pathological corneas, only a few cells were positive for CD34. Although the numbers of CD34-positive cells in the SJS2 corneas were almost normal, the outer part of the stroma lacked CD34-positive cells (fig 2).

\section{Proteoglycan expression in normal and pathological corneas}

Lumican and keratocan are major keratan sulphate proteoglycans that are present in normal corneas ${ }^{19}$ and are produced by keratocytes. In scar tissues, the keratan sulphate proteoglycans are largely replaced by dermatan sulphate proteoglycans, ${ }^{20-22}$ eg biglycan and decorin. To confirm that the keratocytes were abnormal, we examined the change in the composition of the proteoglycan by quantitative real time RT-PCR for lumican, keratocan, and biglycan. The expression of lumican and keratocan varied among individuals and it was not so different from normal corneas. On the other hand, the expression of the messenger RNA of biglycan was higher in all the pathological corneas than in normal corneas. The overexpression of the mRNA of biglycan indicated that corneal keratocytes were not normal.

\section{Immunohistochemistry for inflammatory cells in normal and pathological corneas}

To investigate whether inflammatory cells or antigen presenting cells (APC) were increased in the pathological corneas and to determine their phenotype, immunohistochemistry was performed with several cell surface markers (table 2). CD45positive cells, which were linear-shaped in cross-section, were distributed randomly but were more common in the outer part of the normal corneal stroma (fig 4). ${ }^{23}$ In the pathological corneas, CD45-positive cells were distributed in a more 
Figure 2 Immunohistochemistry of pathological and normal corneas. CD34positive cells can be seen throughout the corneal stroma in the normal cornea. In Stevens-Johnson syndrome (SJS) type 1, ocular cicatricial pemphigoid (OCP) type 1, OCP2, and alkali burned corneas, the number of CD34-positive cells is decreased. In SJS2 corneas, the outer stroma is negative for CD34 (bar $=100 \mu \mathrm{m})$.
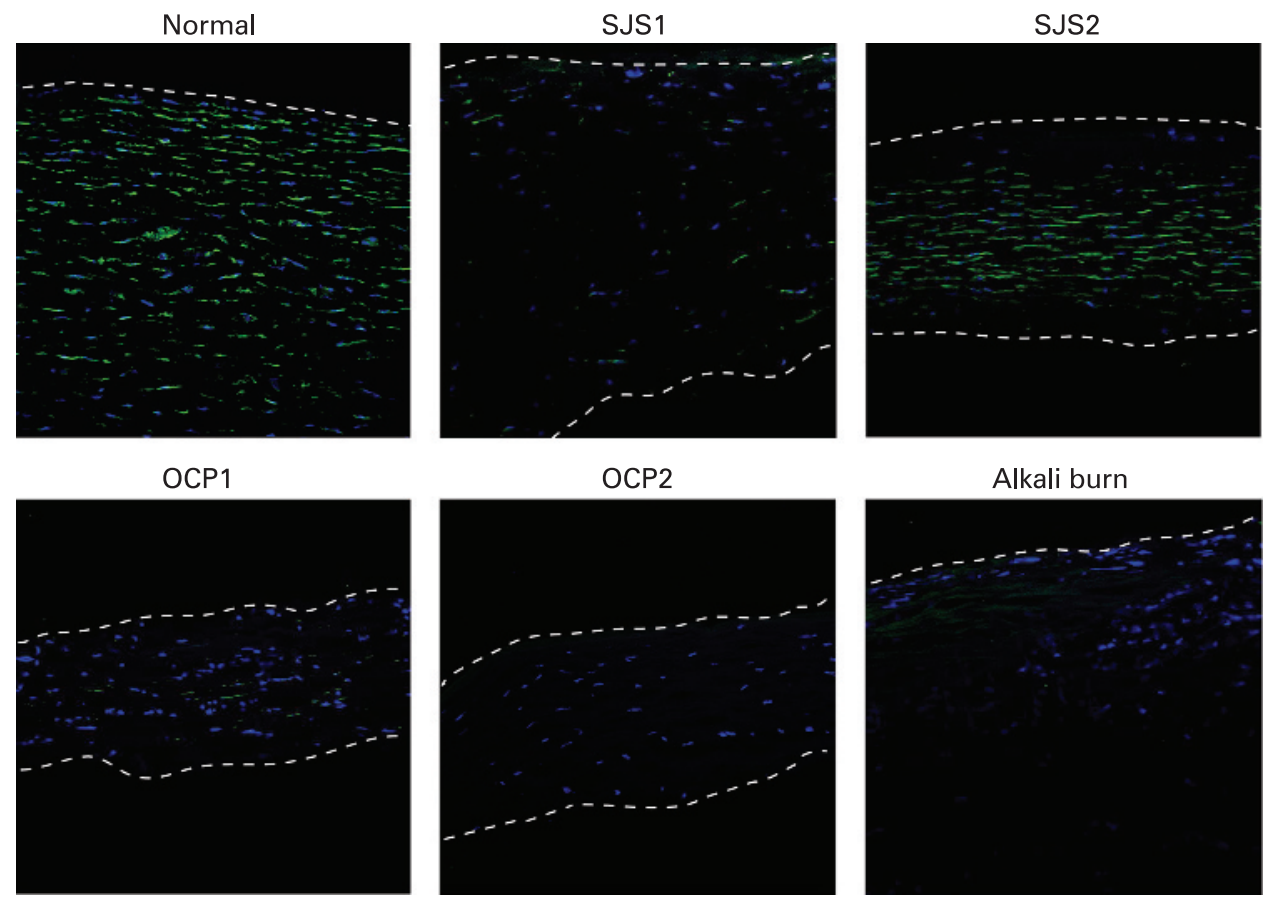

disorganised pattern. In normal corneas, approximately $4-5 \%$ of all cells were CD45 positive (table 3). ${ }^{23}$ Four of the five pathological corneas (except one SJS2) had more CD45-positive cells than normal corneas especially in the OCP1 and alkali burned corneas.

CD14-positive cells were detected more frequently in pathological corneas especially in the SJS1 and alkali burned corneas. Many CD68-positive cells were present in the alkali burned cornea (fig 5). In the SJS1 and OCP corneas, a few cells were positive for CD68, whereas in the SJS2 cornea none of the cells were positive (data not shown). A small number of CD4 and CD8-positive cells was detected in the alkali burned cornea (fig 5), but none was detected in the other corneas. HLA-DRpositive cells were more abundant in the pathological corneas. In the HE-stained OCP1 and alkali burned corneas, some areas appeared to have an infiltration of inflammatory cells. In these parts of the alkali burned cornea, CD45, HLA-DR, CD14, and CD68-positive cells were dominant and a few were CD4 or CD8-positive $\mathrm{T}$ cells (fig 5). CD19-positive B cells were not detected.
From these results, we concluded that macrophages or dendritic cells (DC) were in the majority in areas where inflammatory cells were accumulated. Furthermore, fewer APC were identified in normal than pathological corneas even though they did not appear to contain inflammatory cells.

\section{Chemokine expression in normal and pathological corneas}

We investigated the expression level of chemokines that are chemotactic for monocytes/macrophages and DC. We examined three chemokines: monocyte chemoattractant protein 1 (MCP1), which reacts with the CCR2 receptors expressed on monocytes; macrophage inflammatory protein (MIP) $1 \alpha$, which reacts with receptor CCR1 expressed on monocytes and immature DC; and MIP1 $\beta$, which reacts with receptor CCR5 expressed on monocytes.

The expression of the mRNA of MCP1 was higher in four of the five pathological corneas than in the normal corneas and the expression of MIP1 $\alpha$ and MIP1 $\beta$ was higher in all of the pathological corneas (fig 6). From these results, the expression of the mRNA of chemokines that are chemotactic for
Figure 3 Expression of the mRNA of proteoglycans in normal and pathological corneas. The level of expression of the mRNA of lumican and keratocan is not so different from normal corneas. Biglycan mRNA is expressed at much higher levels in all the pathological corneas. Data in normal corneas are the mean $\pm S D$ ( $n=6)$. GADPH, Glyceraldehyde-3phosphate dehydrogenase; OCP, ocular cicatricial pemphigoid; SJS, StevensJohnson syndrome.
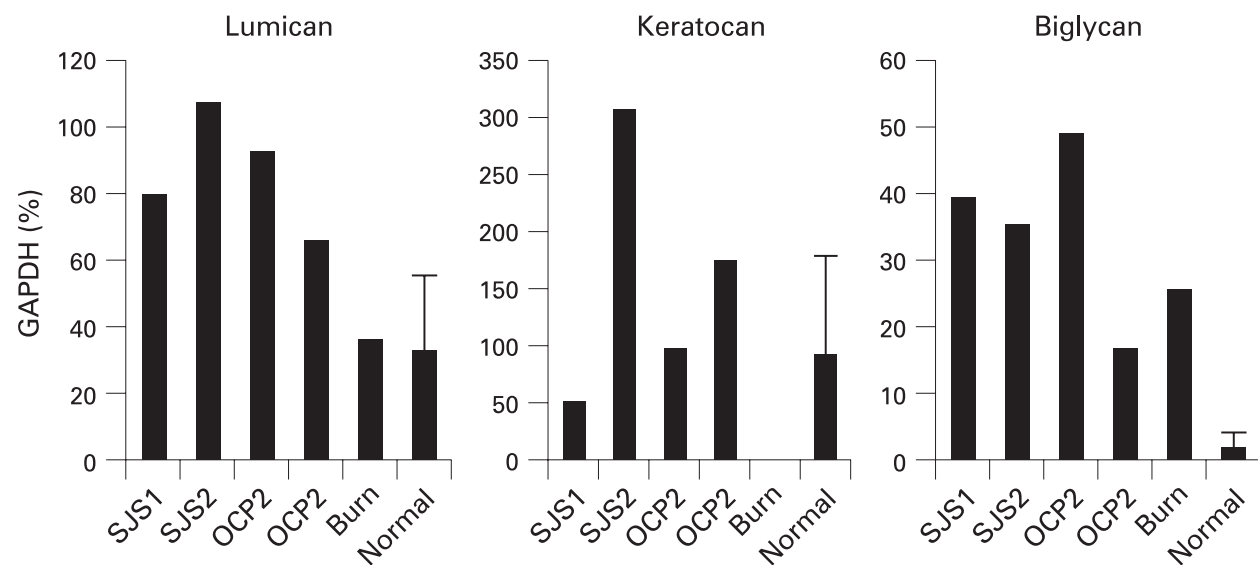
Table 3 Percentage of CD45-positive cells in corneas with different types of pathology

\begin{tabular}{lccc}
\hline & Central & Paracentral & Peripheral \\
\hline SJS1 & 33.4 & 18.2 & 12.9 \\
SJS2 & 3.9 & 3.8 & 5.5 \\
OCP1 & 7.2 & 44.9 & 38.1 \\
OCP2 & 12.6 & 17.5 & 23.1 \\
Alkali burn & 16.0 & 26.2 & 18.7 \\
Normal average $(n=3)$ & 3.9 & 5.1 & 4.5 \\
\hline
\end{tabular}

OCP, Ocular cicatricial pemphigoid; SJS, Stevens-Johnson syndrome.

macrophages/monocytes was higher in the chronic phase in severe ocular surface diseases with stem cell deficiency. This is consistent with the immunohistochemical results that a persistent inflammation is present in the stroma even in the chronic phase.

\section{DISCUSSION}

Our results showed that even in the chronic phase of SJS, OCP, and alkali burn, the number of normal keratocytes is reduced and the number of inflammatory cells and the levels of some chemokines are increased in the corneal stroma.

We used normal corneal buttons of US eye bank eyes as fresh as four to five days postmortem as controls to avoid decreased immunoreactivity for CD45 or CD14. ${ }^{23}$ The corneas of the patients were treated within one hour after excision, but the normal corneas from the United States were used four to five days after excision, so the normal corneas were not an exact match with the experimental corneas. In corneal transplantations, however, donor corneas are frequently used four to five days postmortem and so we believe that any differences in the findings were not caused by the preservation time. To be certain that the time of preservation of corneas was not the cause of changes in the mRNA expression level, we also examined corneas with non-inflammatory stromal opacities ( $n=4)$ processed within one hour after operative excision. These corneas showed similar results to those with inflammatory opacities for proteoglycans, but had lower levels of chemokine mRNA expression (data not shown).

The pathological corneas showed localised or diffuse loss of CD34 expression as reported for corneas with other types of diseases such as keratoconus and herpes keratitis. ${ }^{17}$ The overall decrease in immunoreactivity for CD34 may be caused by the decreased number of keratocytes, although some of the cells with flattened nuclei were considered to be keratocytes and some were CD34 negative. We thus believe that the results reflected the abnormality of the keratocytes as well as a decrease in numbers.

Predictably, the expression of the mRNA of biglycan was increased in all of the pathological corneas because biglycan is reported to increase when corneal fibrosis or scar formation is present. ${ }^{24}$ This change was identified even in SJS1 or SJS2 corneas with mild stromal opacities. The expression of the mRNA of lumican and keratocan varied among individuals and the change might have been caused by individual differences. In any case, the overexpression of biglycan suggested that the keratocytes remained abnormal even in the chronic phase.

Bone marrow-derived APC were recently reported to exist in the stroma of normal corneas. ${ }^{15} 16232526$ These cells are CD45 positive and also positive for CD14, CD11b, CD11c, and HLADR. ${ }^{23}$ Strongly inflamed corneal stroma, such as rejected corneas, were reported to have increased numbers of CD68, CD1a, CD14, HLA-DR, CD4, and CD8-positive cells. ${ }^{27}$ In our
Figure 4 Immunostaining against CD45 and CD14 in normal and pathological corneas. All of the CD14-positive cells (red) are also CD45 positive (green). In the pathological corneas, more cells are positive for CD45 and CD14 than in normal cornea. Magnified images of double positive cells for CD45 and CD14 are also shown (bar $=100 \mu \mathrm{m}$ ). ОCP, Ocular cicatricial pemphigoid; SJS, Stevens-Johnson syndrome.

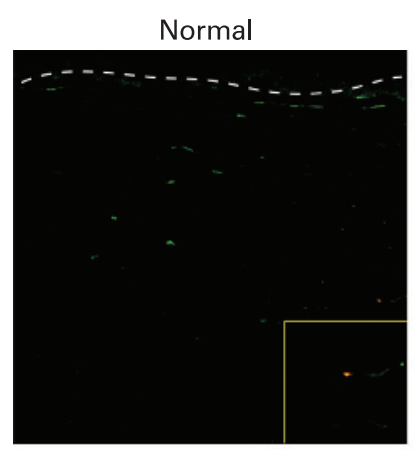

OCP1

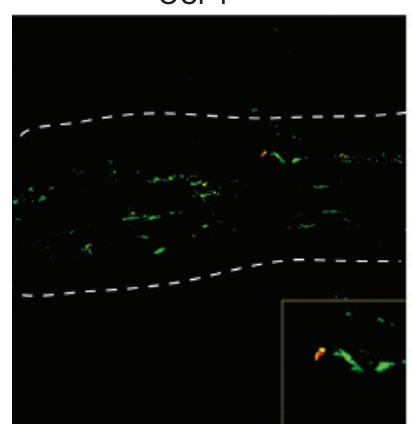

SJS1

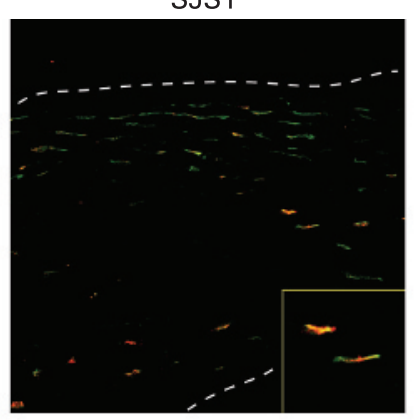

OCP2

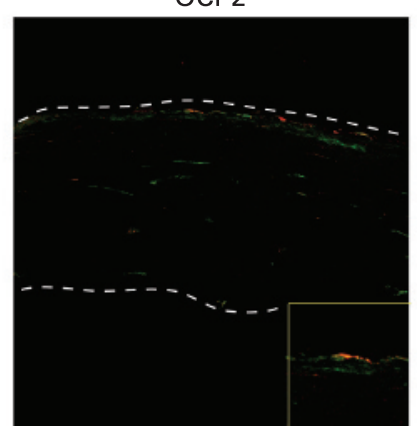

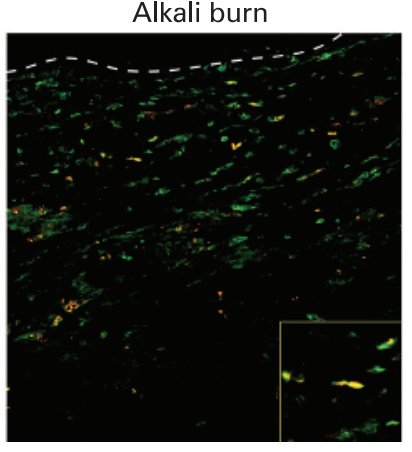


Figure 5 Immunostaining for $\mathrm{CD68}$, CD4, CD8 and HLA-DR of alkali burned corneas. CD68-positive cells are dominant in the area where the inflammatory cells are accumulated. Some of the inflammatory cells are CD4 or CD8 positive. HLA-DR-positive cells can be seen throughout the stroma. Magnified images are also shown (bar $=100 \mu \mathrm{m})$.
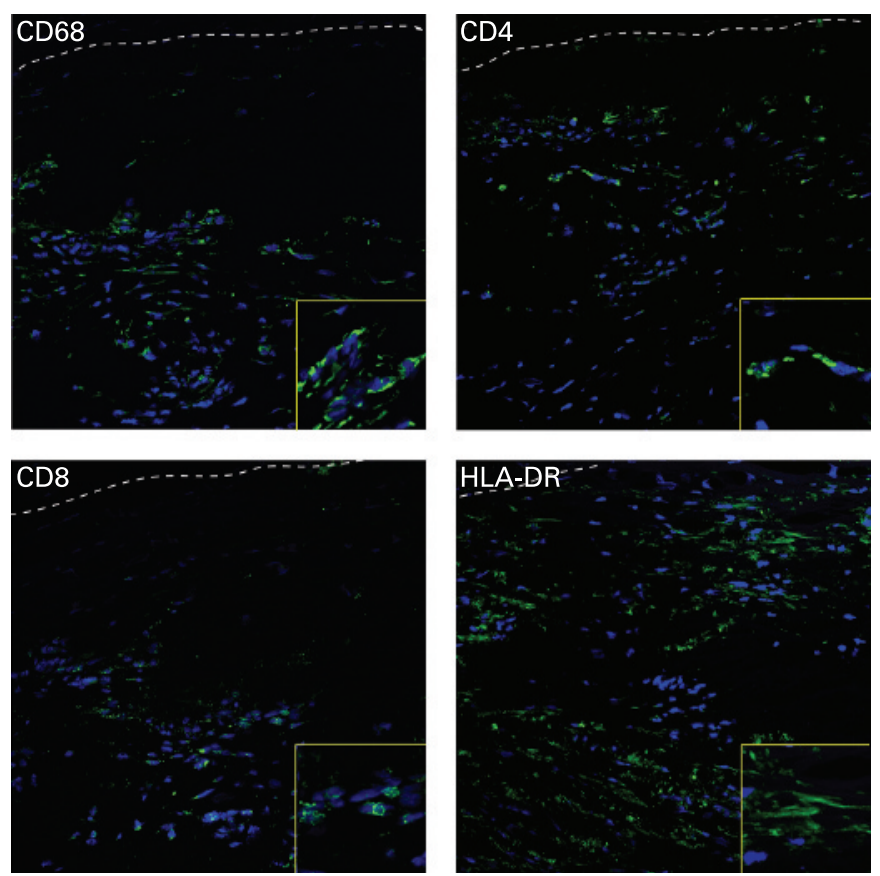

experiment, the number of CD45-positive cells increased in four out of five pathological corneas and CD14, CD68, and HLA-DRpositive cells were also increased. These findings indicated an increase in the number of inflammatory cells, predominantly macrophages and DC.

The level of expression of the mRNA of representative chemokines chemotactic for macrophages/monocytes, especially MIP1 $\alpha$ and MIP1 $\beta$, was higher in the pathological corneas compared with that in normal corneas. Furthermore, the pathological corneas still had markedly higher levels of chemokine mRNA compared with those in corneas with noninflammatory opacities (data not shown). These observations are similar to those in the latent phase of herpes simplex virus 1infected corneas, ${ }^{28}$ in which persistent inflammation was shown to exist. The level of MCP1 was also increased, but was not as elevated as MIP1 $\alpha$ and MIP1 $\beta$. As MCP1 is a potent angiogenic factor, ${ }^{29}$ we believe that the lower degree of upregulation reflects the absence of neovascularisation in the stroma of the pathological corneas.

SJS and OCP are immune mediated whereas alkali burns cause secondary cicatricial keratoconjunctivitis. So alkali burns are pathologically different from SJS or OCP. The results of this experiment, however, showed that the results from the alkali burned corneas were similar to those from the SIS or OCP corneas as reported for conjunctivalised corneal epithelium. ${ }^{14}$ When the conjunctiva completely covers the cornea, it might lead to some abnormalities in the cornea.

In general, when corneal transplantation or other surgeries are performed, it is preferable that the recipient corneas have as little inflammation as possible. ${ }^{30-32}$ We have shown that the corneal stroma of corneas with severe ocular surface diseases with stem cell deficiency have persistent inflammation even in the chronic phase and this should be taken into consideration before surgery because it can cause postoperative inflammation.

In summary, the decreased CD34-positive cells and increased expression of biglycan, increased number of inflammatory cells and APC and increased inflammatory chemokines in the corneal stroma of severe ocular surface diseases with stem cell deficiency suggest that the keratocytes are abnormal and persistent inflammation is present even in the chronic phase. Further experiments with more cases are needed to determine what effect these conditions will have on the corneal epithelium and
Figure 6 Expression of the mRNA of chemokines in normal and pathological corneas. Monocyte chemoattractant protein 1 (MCP1) expression level in pathological corneas is higher than in normal corneas except for StevensJohnson syndrome 2 (SJS2). The level of expression of macrophage inflammatory protein (MIP) $1 \alpha$ and MIP1 $\beta$ is much higher in all the pathological corneas. Data in normal corneas are mean $\pm S D$ ( $n=6$ ). GAPDH, Glyceraldehyde-3phosphate dehydrogenase; OCP, ocular cicatricial pemphigoid; SJS, StevensJohnson syndrome.
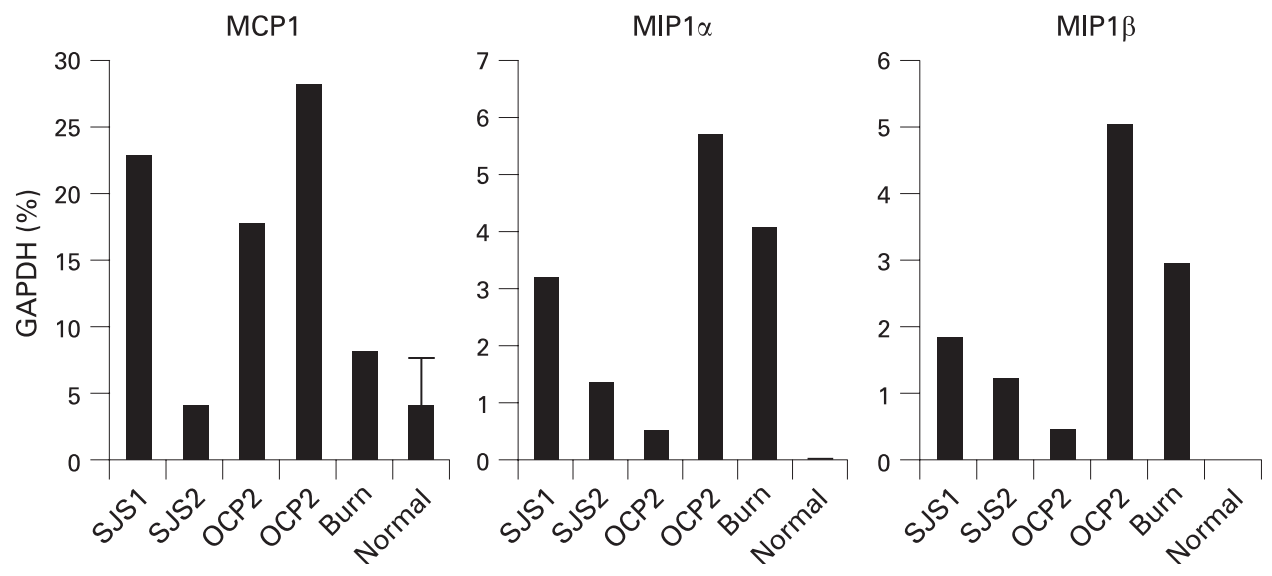
endothelium. More knowledge on keratocytes in the chronic pathological phase will be important to improve operative procedures and the postoperative management of corneal stem cell-deficient diseases.

Competing interests: None declared.

Patient consent: Informed consent was obtained for publication of figure 1.

\section{REFERENCES}

1. Mondino BJ, Brown SI. Immunosuppressive therapy in ocular cicatricial pemphigoid. Am J Ophthalmol 1983;96:453-9.

2. Mondino BJ, Brown Sl. Ocular cicatricial pemphigoid. Ophthalmology 1981;88:95100.

3. Mondino BJ. Cicatricial pemphigoid and erythema multiforme. Ophthalmology 1990;97:939-52

4. Arstikaitis MJ. Ocular aftermath of Stevens-Johnson syndrome. Arch Ophthalmol 1973;90:376-9.

5. Samson CM, Nduaguba C, Baltatzis $\mathrm{S}$, et al. Limbal stem cell transplantation in chronic inflammatory eye disease. Ophthalmology 2002:109:862-8.

6. Shimazaki J, Shimmura S, Fujishima H, et al. Association of preoperative tear function with surgical outcome in severe Stevens-Johnson syndrome. Ophthalmology 2000;107:1518-23.

7. Pfister RR. Chemical injuries of the eye. Ophthalmology 1983;90:1246-53.

8. Brodovsky SC, McCarty CA, Snibson G, et al. Management of alkali burns: an 11year retrospective review. Ophthalmology 2000;107:1829-35.

9. Ilari L, Daya SM. Long-term outcomes of keratolimbal allograft for the treatment of severe ocular surface disorders. Ophthalmology 2002;109:1274-84.

10. Shimazaki J, Aiba M, Goto E, et al. Transplantation of human limbal epithelium cultivated on amniotic membrane for the treatment of severe ocular surface disorders. Ophthalmology 2002;109:1285-90.

11. Tsai RJ, Tseng SC. Human allograft limbal transplantation for corneal surface reconstruction. Cornea 1994;13:389-400.

12. Morgan S, Murray A. Limbal autotransplantation in the acute and chronic phases of severe chemical injuries. Eye 1996:10:349-54.

13. Sangwan VS, Matalia HP, Vemuganti GK, et al. Clinical outcome of autologous cultivated limbal epithelium transplantation. Indian J Ophthalmol 2006:54:29-34.

14. Kawasaki S, Nishida K, Sotozono C, et al. Conjunctival inflammation in the chronic phase of Stevens-Johnson syndrome. Br J Ophthalmol 2000;84:1191-3.
15. Brissette-Storkus CS, Reynolds SM, Lepisto AJ, et al. Identification of a nove macrophage population in the normal mouse corneal stroma. Invest Ophthalmol Vis Sci 2002; 43:2264-71

16. Yamagami S, Usui T, Amano $\mathrm{S}$, et al. Bone marrow-derived cells in mouse and human cornea. Cornea 2005;24(Suppl 1):S71-4.

17. Toti $\mathbf{P}$, Tosi GM, Traversi C, et al. CD-34 stromal expression pattern in normal and altered human corneas. Ophthalmology 2002;109:1167-71.

18. Joseph A, Hossain P, Jham S, et al. Expression of CD34 and L-selectin on human corneal keratocytes. Invest Ophthalmol Vis Sci 2003;44:4689-92.

19. Foster CS, Azar DT, Dohlman CH. Smolin and Thoft's The cornea: scientific foundations and clinical practice, 4th edn. Philadelphia, PA: Lippincott Williams \& Wilkins, 2005;12-13.

20. Hassell JR, Cintron C, Kublin C, et al. Proteoglycan changes during restoration of transparency in corneal scars. Arch Biochem Biophys 1983;222:362-9.

21. Cintron C, Gregory JD, Damle SP, et al. Biochemical analyses of proteoglycans in rabbit corneal scars. Invest Ophthalmol Vis Sci 1990;31:1975-81.

22. Funderburgh JL, Chandlert JW. Proteoglycans of rabbit corneas with noperforating wounds. Invest Ophthalmol Vis Sci 1989;30:435-42.

23. Yamagami S, Ebihara N, Usui T, et al. Bone marrow-derived cells in normal human corneal stroma. Arch Ophthalmol 2006;124:62-9.

24. Funderburgh JL, Hevelone ND, Roth MR, et al. Decorin and biglycan of normal and pathologic human corneas. Invest Ophthalmol Vis Sci 1998;39:1957-64.

25. Hamrah $\mathbf{P}$, Zhang 0 , Liu $Y$, et al. Novel characterization of MHC class II-negative population of resident corneal Langerhans cell-type dendritic cells. Invest Ophthalmol Vis Sci 2002;43:639-46.

26. Brissette-Storkus CS, Reynolds SM, Lepisto AJ, et al. Identification of a novel macrophage population in the normal mouse corneal stroma. Invest Ophthalmol Vis Sci 2002; $43: 2264-71$

27. Kuffova L, Holan V, Lumsden L, et al. Cell subpopulations in failed human cornea grafts. Br J Ophthalmol 2006;83:1364-9.

28. Cook WJ, Kramer MF, Walker RM, et al. Persistent expression of chemokine and chemokine receptor RNAs at primary and latent sites of herpes simplex virus 1 infection. Virol J 2004;1:1-12.

29. Goede V, Brogelli L, Ziche $M$, et al. Induction of inflammatory angiogenesis by monocyte chemoattractant protein-1. Int J Cancer 1999;82:765-70.

30. Yamagami S, Hamrah P, Zhang Q, et al. Early ocular chemokine gene expression and leukocyte infiltration after high-risk corneal transplantation. Mol Vis 2005:11:632-49.

31. Yamada J, Dana MR, Zhu SN, et al. Interleukin 1 receptor antagonist suppresses allosensitization in corneal transplantation. Arch Ophthalmol 1998;116:1351-7.

32. Williams KA, White MA, Ash JK, et al. Leukocytes in the graft bed associated with corneal graft failure. Analysis by immunohistology and actuarial graft survival. Ophthalmology 1989;96:38-44.

\section{Access a vast information database with Toll-Free linking}

"Toll-free" linking gives you immediate access to the full text of many of the cited articles in a paper's reference list-FOR FREE. With the support of HighWire's vast journal catalogue, a huge reference library is now open to you. If HighWire hosts the journal, you can view the full text of the referenced article, completely free of charge by following the Free Full Text links in the references. 